PROCEEDINGS OF THE

AMERICAN MATHEMATICAL SOCIETY

Volume 134, Number 9, September 2006, Pages 2661-2666

S 0002-9939(06)08282-7

Article electronically published on March 23, 2006

\title{
EXTREME CONTRACTIONS ON CONTINUOUS VECTOR-VALUED FUNCTION SPACES
}

\author{
HASAN AL-HALEES AND RICHARD J. FLEMING
}

(Communicated by Joseph A. Ball)

\begin{abstract}
An old question asks whether extreme contractions on $C(K)$ are necessarily nice; that is, whether the conjugate of such an operator maps extreme points of the dual ball to extreme points. Partial results have been obtained. Determining which operators are extreme seems to be a difficult task, even in the scalar case. Here we consider the case of extreme contractions on $C(K, E)$, where $E$ itself is a Banach space. We show that every extreme contraction $T$ on $C(K, E)$ to itself which maps extreme points to elements of norm one is nice, where $K$ is compact and $E$ is the sequence space $c_{0}$.
\end{abstract}

By an extreme contraction, we mean an element $T$ of the set $L(X, Y)$ of bounded linear operators from a Banach space $X$ to a Banach space $Y$, which is an extreme point of the unit ball of $L(X, Y)$. In 1965, Blumenthal, Lindenstrauss, and Phelps [2] showed that the conjugate $T^{*}$ of an extreme contraction $T$ on the space $C(Q)$ of real-valued continuous functions on a compact metric space $Q$ to $C(K)$, where $K$ is compact and Hausdorff, must map the extreme points of $C(K)^{*}$ to extreme points. Such operators have been called nice operators [6]. In fact, what Blumenthal, Lindenstrauss, and Phelps really showed was that the operator $T$ must have the form

$$
T f(t)=h(t) f(\varphi(t)),
$$

where $h \in C(K)$ with modulus one, and $\varphi$ is continuous on $K$ to $Q$. This form is typical of nice operators on continuous function spaces, even in the vector-valued case 1 .

It is always the case, and easy to show, that every nice operator is extreme. The general question to be asked, then, is the converse: if $T \in L(X, Y)$ is extreme, must it be nice? The answer, in general, is no, since it has been shown that there exists a four-dimensional Banach space $X$ and an extreme contraction $T$ from $X$ to $C[0,1]$ that is not nice [2. However, there is a positive answer for certain $C(K)$ spaces, as we have already mentioned above, and other versions of the BLP result have been given by several authors. Utilising results of Sharir [7, Gendler [3] extended the result of BLP [2] to the case of complex-valued functions as follows.

Theorem 1 (Gendler). Let $Q, K$ be compact Hausdorff spaces and let $T$ be an extreme contraction from the space $C(Q)$ of complex-valued functions on $Q$ to the

Received by the editors March 15, 2005 and, in revised form, April 1, 2005.

2000 Mathematics Subject Classification. Primary 47B38, 46E40.

(C)2006 American Mathematical Society 
complex space $C(K)$. Suppose that

(i) the adjoint $T$ maps extreme points of the unit ball of $C(K)^{*}$ to elements of norm one, or

(ii) $K$ is extremally disconnected.

Then $T$ is nice.

A space is extremally disconnected if the closure of every open set is open. An important fact is that in an extremally disconnected space, the space is the StoneCech compactification of every dense subset [4. p. 96].

The papers of Sharir 7 and Gendler 3 provide a good discussion of the history of the general problem and the names of various contributors. Our goal in the present paper is to generalize the result of Theorem 1 to the case of vector-valued continuous functions. The spaces in question are denoted by $C(K, E)$, where $K$ is a compact Hausdorff space and $E$ is a Banach space. We will generally denote an element of $C(K, E)$ by uppercase letters such as $F, G$ and use the lowercase for scalar-valued functions. Thus, for $F \in C(K, E)$, the norm is given by $\|F\|=$ $\sup _{t \in K}\|F(t)\|_{E}$. By $B(X)$ and $S(X)$ we will mean, respectively, the closed unit ball of $X$ and the surface of the unit ball of $X$. The set of extreme points of the unit ball of a Banach space $X$ will be abbreviated by $\operatorname{ext}(X)$. The reader will recall that the elements of the dual space for $C(K, E)$ are regular Borel measures on $K$ with values in $E^{*}$, and the extreme points of the unit ball are the point-mass measures of the form $x^{*} \circ \psi_{t}$, where $x^{*} \in \operatorname{ext}\left(E^{*}\right)$ and $\psi_{t}$ is the evaluation function defined by $\psi_{t}(F)=F(t)$. We will find it convenient to shorten this designation of an extreme point by writing the pair $\left(x^{*}, t\right)$ to represent $x^{*} \circ \psi_{t}$.

The problem of characterizing extreme operators seems difficult. An interesting illustration of this for two-dimensional $\ell^{p}$-spaces can be found in [5. Hence, it may not be surprising that we find it necessary to consider a very narrow class of spaces for $E$. Here is the theorem. The first part of the proof owes much to Gendler's argument in the scalar case.

Theorem 2. Suppose $Q, K$ are compact Hausdorff spaces and $T$ is an extreme contraction on $C(Q, E)$ to $C(K, E)$, where $E=\ell^{\infty}(n)$ or $E=c_{0}$. If $T^{*}$ maps extreme points to elements of norm one, then $T$ is nice.

Proof. For the proof we will assume $E=c_{0}$.

Let us suppose that $T$ is not nice. There are two cases to consider:

(i) there is an extreme point $\left(x^{*}, t\right)$ such that $T^{*}\left(x^{*}, t\right)$ is not a point-mass measure;

(ii) $T^{*}$ maps all extreme points to point-mass measures, but there exists a pair $\left(x^{*}, t\right)$ such that $T^{*}\left(x^{*}, t\right)=\left(y^{*}, s\right)$, where $y^{*}$ is not in $\operatorname{ext}\left(E^{*}\right)$.

Let us begin with (i) We assume then that $T^{*}\left(x^{*}, t\right)$ is a regular Borel measure on $K$ with values in $E^{*}$ and with the norm of the total variation measure $\nu=$ $\left|T^{*}\left(x^{*}, t\right)\right|$ equal to one. The support of this total variation measure, denoted by $\operatorname{supp}\left|T^{*}\left(x^{*}, t\right)\right|=\operatorname{supp} \nu$, is a compact subset of $Q$ with $\nu$ measure 1 and $\nu(D)<1$ for any proper compact subset $D$ of itself. By assumption, there exist $s_{1}, s_{2}$ in supp $\nu$ with $s_{1} \neq s_{2}$. Now there exists a continuous function on $Q$ with range in $[0,1]$ such that $g\left(s_{1}\right)=1$ and $g\left(s_{2}\right)=0$. We claim that $g$ is not constant a.e. with respect to $\nu$. Otherwise, there would exist $A \subset Q$ such that $\nu(A)=0$ and $g=k$ on $A^{c}$ (the complement of $A$ ). One of $s_{1}$ or $s_{2}$ must be in $A$, and the value of $g$ there 
must be different from $k$. If the point is $s_{2}$, then there exists an open neighborhood $V$ of $s_{2}$ with $V \subset A$ and $\nu(V)=0$. Now the union of $V$ and the complement of the support of $\nu$ is an open set with measure zero which contradicts the fact that the complement of the support is the largest open subset of $Q$ with measure zero. This proves our claim.

Next, we define operators $U_{1}, U_{2}$ on $C(Q, E)$ by $U_{1}(F)=T(g F)$ and $U_{2}(F)=$ $T((1-g) F)$. Clearly, these operators are contractions. For each pair $\left(e^{*}, r\right)$ representing an extreme point of $C(K, E)^{*}, T^{*}\left(e^{*}, r\right)$ is a vector measure which is absolutely continuous with respect to its total variation. Since the $\ell^{1}$-spaces satisfy the Radon-Nikodým property, the Radon-Nikodým Theorem guarantees the existence of an integrable function $H\left(e^{*}, r\right)$ such that

$$
T^{*}\left(e^{*}, r\right)(A)=\int_{A} H\left(e^{*}, r\right) d\left|T^{*}\left(e^{*}, r\right)\right| .
$$

This yields the so-called polar decomposition of the measure

$$
d T^{*}\left(e^{*}, r\right)=H\left(e^{*}, r\right) d\left|T^{*}\left(e^{*}, r\right)\right|,
$$

where $\|H\|=1$ a.e. with respect to $\left|T^{*}\left(e^{*}, r\right)\right|$. Moreover, for each $\left(e^{*}, r\right)$ we have

$$
d U_{1}^{*}\left(e^{*}, r\right)=g H\left(e^{*}, r\right) d\left|T^{*}\left(e^{*}, r\right)\right|, \quad d U_{2}\left(e^{*}, r\right)=(1-g) H\left(e^{*}, r\right) d\left|T^{*}\left(e^{*}, r\right)\right| .
$$

Since the norms of the linear functionals determined by the measures are given by the total variation measures of $K$, we have

$$
\begin{aligned}
\left\|U_{1}^{*}\left(e^{*}, r\right)\right\|+\left\|U_{2}^{*}\left(e^{*}, r\right)\right\| & =\int_{K}\left\|g H\left(e^{*}, r\right)\right\| d\left|T^{*}\left(e^{*}, r\right)\right| \\
& +\int_{K}\left\|(1-g) H\left(e^{*}, r\right)\right\| d\left|T^{*}\left(e^{*}, r\right)\right| \\
& =\int_{K} g d\left|T^{*}\left(e^{*}, r\right)\right|+\int_{K}(1-g) d\left|T^{*}\left(e^{*}, r\right)\right| \\
& =\int_{K} d\left|T^{*}\left(e^{*}, r\right)\right|=1 .
\end{aligned}
$$

Let us now define $V$ on the extreme points of $C(K, E)^{*}$ by

$$
V\left(e^{*}, r\right)=\left\|U_{2}^{*}\left(e^{*}, r\right)\right\| U_{1}^{*}\left(e^{*}, r\right)-\left\|U_{1}^{*}\left(e^{*}, r\right)\right\| U_{2}^{*}\left(e^{*}, r\right) .
$$

We define a bounded operator $U$ on $C(Q, E)$ by

$$
e^{*}(U F(r))=V\left(e^{*}, r\right)(F)
$$

for each $e^{*} \in \operatorname{ext}\left(E^{*}\right)$ and $r \in K$. Note that since there is a Schauder basis for $E^{*}=\ell^{1}$ whose modulus one scalar multiples exhaust the entire collection of extreme points of $B\left(E^{*}\right)$, the above equation really does define $U$. Then $\left.U^{*}\left(e^{*}, r\right)\right)=$ $V\left(e^{*}, r\right)$ for each $\left(e^{*}, r\right)$, and we have

$$
\begin{aligned}
\left\|(T \pm U)^{*}\left(e^{*}, r\right)\right\| & =\left\|\left(1 \pm\left\|U_{2}^{*}\left(e^{*}, t\right)\right\|\right) U_{1}^{*}\left(e^{*}, r\right)+\left(1 \mp\left\|U_{1}^{*}\left(e^{*}, r\right)\right\|\right) U_{2}^{*}\left(e^{*}, r\right)\right\| \\
& \leq\left\|U_{1}^{*}\left(e^{*}, r\right)\right\|+\left\|U_{2}^{*}\left(e^{*}, r\right)\right\|=1 .
\end{aligned}
$$

Suppose that $F \in C(K, E)$ with $\|F\|=1$. There exists an extreme point $\left(e^{*}, r\right)$ (one for + and one for - ) such that

$$
\|(T \pm U) F\|=\left|e^{*}(T \pm U)(F)(r)\right|=\left|(T \pm U)^{*}\left(e^{*}, r\right)(F)\right| \leq 1 .
$$

It follows that $\|T \pm U\| \leq 1$, and since $T$ is extreme, we must have $U=0$. This assumption would lead us to conclude that for every pair $\left(e^{*}, r\right), g$ is constant 
a.e. with respect to the total variation measure $\left|T^{*}\left(e^{*}, r\right)\right|$. However, we observed above that this is not the case for the measure $\nu=\left|T^{*}\left(x^{*}, t\right)\right|$, and this contradiction proves that $T$ cannot satisfy the first case.

Next we turn to (ii) First we argue that given any $y^{*} \in B\left(E^{*}\right)$, we can write $y^{*}$ as a unique convex combination of two elements of $B\left(E^{*}\right)$, one of which has at most finitely many nonzero entries. To this end, let $y^{*}=\left(a_{1}, a_{2}, \ldots\right) \neq 0$ be given, and choose the positive integer $k$ with the property that $\sum_{j=1}^{k}\left|a_{j}\right| \geq \frac{1}{2}\left\|y^{*}\right\|$ while $\sum_{j=1}^{k-1}\left|a_{j}\right|<\frac{1}{2}\left\|y^{*}\right\|$. If $a_{k}=e^{i \theta}\left|a_{k}\right|$, let $b_{k}=e^{i \theta}\left(\left\|y^{*}\right\|-2 \sum_{j=1}^{k-1}\left|a_{j}\right|\right)$. Define

$$
\begin{aligned}
& y_{1}^{*}=\left(2 a_{1}, 2 a_{2}, \ldots, 2 a_{k-1}, b_{k}, 0,0, \ldots\right), \\
& y_{2}^{*}=\left(0,0, \ldots, 0,2 a_{k}-b_{k}, 2 a_{k+1}, 2 a_{k+2}, \ldots\right) .
\end{aligned}
$$

Then $\left\|y_{1}^{*}\right\|=\left\|y_{2}^{*}\right\|=\left\|y^{*}\right\|$ and

$$
y^{*}=\frac{1}{2} y_{1}^{*}+\frac{1}{2} y_{2}^{*}
$$

as advertised. If $y^{*}=0$, let $y_{1}^{*}=e_{1}^{*}, y_{2}^{*}=-e_{1}^{*}$.

Now suppose that $T\left(e^{*}, t\right)=\left(y^{*}, s(t)\right)$, where $y^{*} \notin \operatorname{ext}\left(E^{*}\right)$. For each $r \in K$ we have

$$
T^{*}\left(e^{*}, r\right)=\left(y_{r}^{*}, s(r)\right)
$$

where $y_{r}^{*} \in B\left(E^{*}\right)$ and $s(r) \in Q$. We define $V$ on the extreme points of $C(K, E)^{*}$ by

$$
V\left(z^{*}, r\right)= \begin{cases}\left(\frac{1}{2} y_{r, 1}^{*}-\frac{1}{2} y_{r, 2}^{*}, s(r)\right) & \text { if } z^{*}=e^{*} \\ 0 & \text { otherwise. }\end{cases}
$$

Here, $y_{r, 1}^{*}$ and $y_{r, 2}^{*}$ are the unique elements associated with $y_{r}^{*}$ given in (2) and (3). It is important to note that $V\left(e^{*}, r\right) \neq 0$ if $y^{*}$ is not an extreme point. We use this $V$ to define a linear operator $U$ on $C(Q, E)$ to $C(K, E)$ in a way similar to what we did in the first case. Given $F \in C(Q, E), r \in K$, and $z^{*} \in \operatorname{ext}\left(E^{*}\right)$ we define

$$
z^{*}(U F(r))=V\left(z^{*}, r\right)(F) .
$$

We need to see that $U F$ really is an element of $C(K, E)$. We observe that the only nonzero coordinate of $U F(r)$ for any $r \in K$ is the one corresponding to the extreme point $e^{*}$. Hence, to show that $U F$ is continuous at $r$, it suffices to show that given any net $\left\{r_{\alpha}\right\}$ in $K$ converging to $r$, we must have $e^{*}\left(U F\left(r_{\alpha}\right)\right) \rightarrow e^{*}(U F(r))$.

Since $T F\left(r_{\alpha}\right) \rightarrow T F(r)$, we have $T^{*}\left(e^{*}, r_{\alpha}\right)$ converging in the weak*-topology to $T^{*}\left(e^{*}, r\right)$, and as a consequence, $y_{r_{\alpha}}^{*}$ converges coordinatewise to $y_{r}^{*}$. Because of this it will be true that for some index $\alpha_{0}$, the elements $y_{r_{\alpha}, 1}^{*}$ and $y_{r, 1}^{*}$ as given by (2) for $\alpha \geq \alpha_{0}$, will have zero values for all coordinates beyond some fixed $k$. Hence, by the construction given in (2) and the coordinatewise convergence, the corresponding net will converge to $y_{r, 1}^{*}$ in the weak ${ }^{*}$-topology of $E^{*}$. Therefore, we also have $y_{r_{\alpha}, 2}^{*} \rightarrow y_{r, 2}^{*}$. Furthermore, we must have $s\left(r_{\alpha}\right) \rightarrow s(r)$ in $Q$. Suppose not. Then there is a neighborhood $A$ of $s(r)$ and a subnet $s\left(r_{\beta}\right)$ such that $s\left(r_{\beta}\right) \notin A$ for every $\beta$. Let $g$ be a continuous function on $Q$ to $[0,1]$ which is zero on $Q \backslash A$ and one at $s(r)$. Let $u \in E$ be such that $y_{r}^{*}(u) \neq 0$ and $G_{0}$ is the function that is constantly $u$. If $G=g G_{0}$, then

$$
0=y_{r_{\beta}}^{*}\left(G\left(s\left(r_{\beta}\right)\right)\right) \nrightarrow y_{r}^{*}(G(s(r))) \neq 0 .
$$

From these considerations, it is now easy to see that $U^{*}\left(e^{*}, r_{\alpha}\right)(F) \rightarrow U^{*}\left(e^{*}, r\right)(F)$ for all $F \in C(Q, E)$, which is what we needed to show. 
Finally, we see that

$$
\left|\left(T^{*} \pm U^{*}\right)\left(z^{*}, r\right)(F)\right|=\left|y_{r, j}^{*}(F(s(r)))\right| \leq\|F\|,
$$

where $j=1,2$ corresponding to,+- respectively. It follows in the same way as in the proof of case (i) that $\|T \pm U\| \leq 1$. However, since $y^{*}$ is not extreme, $U^{*}\left(e^{*}, t\right) \neq$ 0 so that $U \neq 0$, which is a contradiction of the fact that $T$ is extreme.

NOTE: In the above proof, we have treated the case where $E=c_{0}$. If $E=\ell^{\infty}(n)$, the proof of case (i) is not affected, and in case (ii) the same decomposition of $y_{r}^{*}$ can be carried out, but there are only finitely many coordinates. Thus, the convergence of the net $\left\{y_{r_{\alpha}, 1}^{*}\right\}$ to $y_{r, 1}^{*}$ is clear.

It would be nice to be able to remove the hypothesis about $T^{*}$ mapping extreme points to elements of norm one. We can do so by restricting $K$ as in Theorem 1 . part (ii). First we adapt a result of Sharir $[3$ to our situation.

Lemma 3. Let $T$ be an extreme contraction as in the statement of Theorem 2, For each $e^{*} \in \operatorname{ext}\left(E^{*}\right)$, the set

$$
A\left(e^{*}\right)=\left\{t \in K:\left\|T^{*}\left(e^{*}, t\right)\right\|=1\right\}
$$

is dense in $K$.

Proof. Let $e^{*}$ be given and fixed. For each positive integer $n$ let $G_{n}=\{t \in K$ : $\left.\left\|T^{*}\left(e^{*}, t\right)\right\|>1-1 / n\right\}$. It follows that this set is open in $K$. We claim that it is also dense. To see this, suppose that $f$ is a continuous function on $K$ into $[0,1]$ with $f=0$ on $G_{n}$. Let $\mu$ be a norm-one element of $C(Q, E)^{*}$ and define $V$ on $\operatorname{ext}\left(C(K, E)^{*}\right)$ by

$$
V\left(e^{*}, t\right)=(1 / n) f(t) \mu \text { for } t \in K,
$$

and $V\left(x^{*}, t\right)=0$ for all $x^{*} \in \operatorname{ext}\left(E^{*}\right)$ different from $e^{*}$ and all $t \in K$. Note that $\left\|T^{*}\left(e^{*}, t\right)-V\left(e^{*}, t\right)\right\| \leq 1$. As we did in the proof of Theorem 2, we define an operator $U$ on $C(Q, E)$ by

$$
x^{*}(U F)(t)=V\left(e^{*}, t\right)(F)
$$

for $t \in K$ and $x^{*} \in \operatorname{ext}\left(E^{*}\right)$. Then $U^{*}\left(x^{*}, t\right)=V\left(x^{*}, t\right)$, and for any $F \in C(Q, E)$ with $\|F\|=1$, there exists an extreme point $\left(x^{*}, t\right)$ in the dual such that

$$
\|(T \pm U)(F)\|=\left|x^{*}(U F(t))\right|=\left|\left(T^{*} \pm U^{*}\right)\left(x^{*}, t\right)\right| \leq 1 .
$$

Thus $\|T \pm U\| \leq 1$ and $T$ extreme implies that $U=0$. From this we see that $(1 / n) f(t) \mu=0$ for all $t \in K$. Hence $f=0$, and we conclude that $G_{n}$ is dense. It follows from the Baire Category Theorem that

$$
A\left(e^{*}\right)=\bigcap_{n=1}^{\infty} G_{n}
$$

is also dense.

A slight modification of the proof of Theorem 2 now yields the following.

Theorem 4. Suppose $K$ is an extremally-disconnected compact Hausdorff space and $T$ is an extreme contraction on $C(Q, E)$ to $C(K, E)$, where $E=\ell^{\infty}(n)$ or $E=c_{0}$. Then $T$ is nice. 
Proof. We proceed exactly as in the case of the proof of Theorem 2 If we let $\nu_{1}\left(e^{*}, r\right)=\left\|U_{1}^{*}\left(e^{*}, r\right)\right\|$ and $\nu_{2}\left(e^{*}, r\right)=\left\|U_{2}^{*}\left(e^{*}, r\right)\right\|$ for $r \in A\left(e^{*}\right)$ (as in the lemma), it can be shown that these functions are continuous on $A\left(e^{*}\right)$. Since $K$ is extremally disconnected, it is the Stone-Cech compactification of the dense subset $A\left(e^{*}\right)$, so $\nu_{1}$ and $\nu_{2}$ can be extended continuously to all of $K$ and so that $\nu_{1}\left(e^{*}, r\right)+\nu_{2}\left(e^{*}, r\right)=1$ for all $r \in K$. Hence equation (11) can be extended to all of $K$. The rest of the argument follows as before. Note that the proof of case (ii) in Theorem 2 did not require the assumption about mapping extreme points to elements of norm one.

\section{REFERENCES}

[1] H. Al-Halees and R. Fleming, Extreme point methods and Banach-Stone Theorems, J. Aust. Math. Soc. 75 (2003), 125-143. MR1984631 (2004g:46017)

[2] R. Blumenthal, J. Lindenstrauss, and R. Phelps, Extreme operators into $C(K)$, Pacific J. Math. 15 (1965), 747-756. MR0209862 (35:758)

[3] A. Gendler, Extreme operators in the unit ball of $L(C(X), C(Y))$ over the complex field, Proc. Amer. Math. Soc. 57 (1976), 85-88. MR0405173 (53:8967)

[4] L. Gillman and M. Jerison, Rings of continuous functions, Van Nostrand, Princeton, 1960. MR0116199 (22:6994)

[5] R. Grzaslewicz, Extreme operators on 2-dimensional $\ell_{p}$-spaces, Colloq. Math. 24 (1981), 309315. MR0652589 (83i:47039)

[6] P. Morris and R. Phelps, Theorems of Krein-Milman type for certain convex sets of operators, Trans. Amer. Math. Soc. 150 (1970), 183-200. MR0262804 (41:7409)

[7] M. Sharir, Characterizations and properties of extreme operators into $C(Y)$, Israel J. Math. 12 (1972), 174-183. MR.0317026 (47:5574)

Department of Mathematics, Saginaw Valley State University, University Center, Michigan 48710-0001

Department of Mathematics, Central Michigan University, Mt. Pleasant, Michigan 48859 